\title{
Transverse intersections between invariant manifolds of doubly hyperbolic invariant tori, via the Poincaré-Mel'nikov method
}

\author{
Amadeu Delshams ${ }^{1}$, Pere Gutiérrez ${ }^{1}$, Oksana Koltsova $^{2}$, Juan R. Pacha ${ }^{1}$ \\ 1 Dep. de Matemàtica Aplicada I, Universitat Politècnica de Catalunya \\ Av. Diagonal 647, 08028 Barcelona, Catalonia, Spain \\ amadeu.delshams@upc.edu, pere.gutierrez@upc.edu, juan.ramon.pacha@upc.edu \\ 2 Department of Mathematics, Imperial College London \\ SW7 2AZ London, UK \\ o.koltsova@imperial.ac.uk
}

December 22, 2009

\begin{abstract}
We consider a perturbation of an integrable Hamiltonian system having an equilibrium point of elliptichyperbolic type, having a homoclinic orbit. More precisely, we consider an $(n+2)$-degree-of-freedom near integrable Hamiltonian with $n$ centres and 2 saddles, and assume that the homoclinic orbit is preserved under the perturbation. On the centre manifold near the equilibrium, there is a Cantorian family of hyperbolic KAM tori, and we study the homoclinic intersections between the stable and unstable manifolds associated to such tori. We establish that, in general, the manifolds intersect along transverse homoclinic orbits. In a more concrete model, such homoclinic orbits can be detected, in a first approximation, from nondegenerate critical points of a Mel'nikov potential. We provide bounds for the number of transverse homoclinic orbits using that, in general, the potential will be a Morse function (which gives a lower bound) and can be approximated by a trigonometric polynomial (which gives an upper bound).
\end{abstract}

\section{Introduction and setup}

We consider an analytic perturbed Hamiltonian

$$
H=H^{(0)}+\varepsilon H^{(1)},
$$

with $n+2$ degrees of freedom, and $\varepsilon$ as a small parameter, having an equilibrium point with $n$ centres and 2 saddles, i.e. the equilibrium point has $2 n$ elliptic directions and 4 hyperbolic directions. Under suitable conditions, applying KAM theorem on the centre manifold we have a Cantorian family of $n$-dimensional invariant tori, with stable and unstable invariant manifolds, both $(n+2)$-dimensional. Assuming that the equilibrium point has a homoclinic orbit, our aim is to give conditions for the existence of transverse intersections (i.e. transverse homoclinic orbits) between the stable and unstable manifolds of a given invariant torus.

In this paper, we present the main ideas underlying this problem and, under some hypotheses (see below), we show that transverse intersections can be detected as nondegenerate critical points of a periodic function of $n$ angles called the Mel'nikov potential. This generalizes the case of 2 centres and 1 saddle, previously studied in [KLDG05]. Although the expression (12) of the Mel'nikov potential is analogous to the one obtained there, increasing the number of saddles requires a somewhat more complicated approach, that takes into account the additional hyperbolic directions. 
In order to give a more precise description and present our hypotheses, we consider symplectic coordinates

$$
(x, y, q, p)=\left(x_{1}, \ldots, x_{n}, y_{1}, \ldots, y_{n}, q_{1}, q_{2}, p_{1}, p_{2}\right) \in \mathbb{R}^{2 n+4},
$$

where the coordinates $(x, y)$ and $(q, p)$ correspond to the elliptic and hyperbolic directions, respectively. Often, we shall replace $(x, y)$ by the symplectic polar coordinates $(\varphi, \xi)$ (angles and actions), defined by

$$
x_{j}=\sqrt{2 \xi_{j}} \sin \varphi_{j}, \quad y_{j}=\sqrt{2 \xi_{j}} \cos \varphi_{j}, \quad j=1, \ldots, n,
$$

where $\xi_{j}=\left(x_{j}^{2}+y_{j}^{2}\right) / 2$.

The Hamiltonian equations associated to (1) are

$$
\begin{array}{ll}
\dot{x}=\partial_{y} H(x, y, q, p), & \dot{q}=\partial_{p} H(x, y, q, p), \\
\dot{y}=-\partial_{x} H(x, y, q, p), & \dot{p}=-\partial_{q} H(x, y, q, p) .
\end{array}
$$

Let us describe in more detail the Hamiltonian considered, and present the organization of the paper. We assume for $H$ the following hypotheses:

(A) In the unperturbed Hamiltonian, the centre and saddle parts can be separated:

$$
H^{(0)}(x, y, q, p)=F(x, y)+P(q, p) \text {. }
$$

(B) The $n$-d.o.f. Hamiltonian $F$ is an $n$-centre and it is integrable, depending only on the actions:

$$
F(x, y)=f(\xi)=\langle\omega, \xi\rangle+\frac{1}{2}\langle\Omega \xi, \xi\rangle+\mathcal{O}_{3}(\xi)
$$

Thus, the origin of $F$ is an elliptic equilibrium point, with the frequency vector $\omega=\left(\omega_{1}, \ldots, \omega_{n}\right)$ (or the characteristic exponents $\left.\pm \mathrm{i} \omega_{1}, \ldots, \pm \mathrm{i} \omega_{n}\right)$. We assume nonresonance up to order four, $\langle k, \omega\rangle \neq 0$ for all $0<$ $|k|_{1} \leq 4$, and one of the nondegeneracy conditions: $\operatorname{det} \Omega \neq 0$ or $\operatorname{det}\left(\begin{array}{cc}\Omega & \omega \\ \omega^{\top} & 0\end{array}\right) \neq 0$.

(C) The 2-d.o.f. Hamiltonian $P$ is an integrable 2-saddle, with real and simple characteristic exponents $\pm \lambda_{1}= \pm 1$ and $\pm \lambda_{2}= \pm \lambda$, with $\lambda_{1}>\lambda_{2}>0$. Thus, the origin of $P$ is a hyperbolic equilibrium point. We assume that the additional first integral $K$ is such that the respective quadratic parts $P^{(2)}, K^{(2)}$ are independent functions (excluding the origin). Besides, we assume that $P$ has a homoclinic orbit or loop $\gamma$, biasymptotic to the origin along the strong stable/unstable directions, i.e. associated to the exponents $\pm \lambda_{1}$.

(D) The perturbation $H^{(1)}$ is such that the Hamiltonian $H=H^{(0)}+\varepsilon H^{(1)}$ has the origin as an equilibrium point, with a loop $\gamma_{\varepsilon}$ inherited from $\gamma$, i.e. tangent to the strong stable/unstable directions of the origin. Besides, we assume that $\partial_{q} H^{(1)}=\partial_{p} H^{(1)}=0$ for $q=p=0$.

Let us see what these hypotheses imply. First, we see from (B) that all orbits of $F$ lie in $n$-dimensional invariant tori $\mathcal{T}_{I, 0}=\{\xi=I\}$, for any fixed $I=\left(I_{1}, \ldots, I_{n}\right)$. Each torus has inner dynamics $\dot{\varphi}=\widetilde{\omega}(I)$, with the frequency vector

$$
\widetilde{\omega}(I)=\partial_{\xi} f(I)=\omega+\Omega I+\mathcal{O}_{2}(I) .
$$

The additional conditions in (B) are required in order to apply KAM theorem to a perturbation of $F$ (see below).

Concerning (C), one can carry out a symplectic linear change $(\hat{u}, \hat{v}) \mapsto(q, p)$ such that the quadratic part $P^{(2)}(q, p)$ becomes $\widehat{P}^{(2)}(\hat{u}, \hat{v})=\lambda_{1} \hat{u}_{1} \hat{v}_{1}+\lambda_{2} \hat{u}_{2} \hat{v}_{2}$. For the first integral $K(q, p)$, its quadratic part takes an analogous form: $\widehat{K}^{(2)}(\hat{u}, \hat{v})=\mu_{1} \hat{u}_{1} \hat{v}_{1}+\mu_{2} \hat{u}_{2} \hat{v}_{2}$ (see [LU98, §2.1]). Then, the independence condition is $\lambda_{1} \mu_{2}-\lambda_{2} \mu_{1} \neq 0$. Under this condition, we can apply Vey's result [Vey78], which establishes that there exists a symplectic transformation close to the identity,

$$
(\hat{u}, \hat{v})=\phi(u, v)=(u, v)+\mathcal{O}_{2}(u, v)
$$


analytic (convergent) in a neighbourhood of the origin, such that both the transformed Hamiltonian $\widetilde{P}=\widehat{P} \circ \phi$ and the additional integral $\widetilde{K}=\widehat{K} \circ \phi$ are analytic functions of the products

$$
\eta_{1}:=u_{1} v_{1}, \quad \eta_{2}:=u_{2} v_{2}
$$

In particular, this yields

$$
\widetilde{P}(u, v)=\lambda_{1} \eta_{1}+\lambda_{2} \eta_{2}+\Xi\left(\eta_{1}, \eta_{2}\right)=\eta_{1}+\lambda \eta_{2}+\Xi\left(\eta_{1}, \eta_{2}\right)
$$

with $\Xi\left(\eta_{1}, \eta_{2}\right)=\mathcal{O}_{2}\left(\eta_{1}, \eta_{2}\right)$, in a neighbourhood the origin (see also [Ito89], and [LU98, §2.2] for an account of related results). In the normal form variables, the 2 -dimensional unstable manifold $W_{0}^{\mathrm{u}}$ is given locally by $v_{1}=v_{2}=0$, with Lyapunov exponents $\lambda_{1}, \lambda_{2}$, and the 2 -dimensional stable manifold $W_{0}^{\mathrm{s}}$ is given locally by $u_{1}=u_{2}=0$, with Lyapunov exponents $-\lambda_{1},-\lambda_{2}$. The loop $\gamma$, contained in both manifolds, is given locally by $\eta_{2}=u_{2} v_{2}=0$. It is asymptotic to the origin along the directions associated to the exponents $\pm \lambda_{1}$, which are the strong ones.

We see from $(\mathbf{A}-\mathbf{C})$ that the unperturbed Hamiltonian $H^{(0)}$ is completely integrable, and the following simple facts are easily verified:

* There is a loop $\{0\} \times \gamma$, biasymptotic to the origin, and we denote it again as $\gamma$.

* There is an $n$-parameter set of $n$-dimensional hyperbolic tori $\mathcal{T}_{I, 0} \times\{0\}$, that we denote again as $\mathcal{T}_{I, 0}$. Such tori are contained in the $2 n$-dimensional centre manifold $\mathcal{W}^{c}=\mathbb{R}^{2 n} \times\{0\}$.

* Each torus $\mathcal{T}_{I, 0}$ has $(n+2)$-dimensional stable and unstable manifolds $\mathcal{W}_{I, 0}^{\mathrm{s}, \mathrm{u}}=\mathcal{T}_{I, 0} \times W_{0}^{\mathrm{s}, \mathrm{u}}$, which intersect nontransversely along the homoclinic cylinder $\mathcal{T}_{I, 0} \times \gamma$. Such manifolds $\mathcal{W}_{I, 0}^{\mathrm{s}, \mathrm{u}}$ are contained in the $(2 n+2)$-dimensional centre-stable and centre-unstable manifolds $\mathcal{W}_{0}^{\mathrm{cs}, \mathrm{cu}}=\mathbb{R}^{2 n} \times W_{0}^{\mathrm{s}, \mathrm{u}}$.

The conditions in (D) concern the perturbation $H^{(1)}$. We are assuming that the origin is kept as an equilibrium point, and that $\mathcal{W}^{\mathrm{c}}$ is kept as the centre manifold (due to that $\partial_{q} H_{1}=\partial_{p} H_{1}=0$ for $q=p=0$ ). This allows us to fix ideas, although it is not strictly necessary (see also [KLDG05]).

The inner flow on $\mathcal{W}^{c}$ is given by a perturbed Hamiltonian $F_{\varepsilon}(x, y)=F(x, y)+\varepsilon H^{(1)}(x, y, 0,0)$. The conditions in (B) are the usual for KAM theorem near an elliptic equilibrium point (see for instance [Pös82]), and imply that for $\varepsilon$ small enough the Hamiltonian $F_{\varepsilon}(x, y)$ has a Cantorian ( $n$-parametric) set of $n$-dimensional invariant tori around this point. More precisely, there is a symplectic change of coordinates $(\theta, I) \mapsto(x, y)$, depending on $\varepsilon$, such that $F_{\varepsilon}(x, y)=f_{\varepsilon}(\theta, I)$ satisfies that $\partial_{\theta} f_{\varepsilon}$ vanishes on a Cantorian set $\mathcal{D}$ of $I$, of positive measure near the origin of $F$. The new coordinates $(\theta, I)$ can be taken $\mathcal{O}(\varepsilon)$-close to the symplectic polar coordinates $(\varphi, \xi)$ defined in $(2)$. The set $\mathcal{D}$ corresponds to the actions $I$ such that their frequencies (5) satisfy a Diophantine condition. For $I \in \mathcal{D}$, the set $\mathcal{T}_{I, \varepsilon}=\{I=$ const $\}$ is an $n$-dimensional invariant torus, with a frequency vector $\widetilde{\omega}_{\varepsilon}(I)=\partial_{I} f_{\varepsilon}$.

The tori $\mathcal{T}_{I, \varepsilon}$ are of hyperbolic type when considered in the full phase space, with stable and unstable manifolds $\mathcal{W}_{I, \varepsilon}^{\mathrm{s}, \mathrm{u}}$ that remain close to the unperturbed ones $\mathcal{W}_{I, 0}^{\mathrm{s}, \mathrm{u}}$. Nevertheless, in general the perturbed manifolds do not intersect anymore along a non-transverse "cylinder" (i.e. a set homeomorphic to $\mathbb{T}^{n} \times \mathbb{R}$ ). We are going to show in Section 2 that, under the hypothesis that the loop $\gamma$ is preserved, they intersect generically along a finite number of transverse homoclinic orbits.

The preservation of the loop $\gamma$ is necessary for the existence of homoclinic orbits asymptotic to the KAM tori $\mathcal{T}_{I, \varepsilon}$ that are very close to the origin (i.e. for $|I|$ very small). Indeed, if the perturbed stable and unstable branches of the loop, $\gamma_{\varepsilon}^{\mathrm{s}, \mathrm{u}}$, do not coincide, the stable and unstable manifolds of such tori, $\mathcal{W}_{I, \varepsilon}^{\mathrm{s}, \mathrm{u}}$ do not intersect since they remain very close to $\gamma_{\varepsilon}^{\mathrm{s}, \mathrm{u}}$ (however, for tori $\mathcal{T}_{I, \varepsilon}$ not so close to the origin, i.e. for $|I|$ larger, there may be intersections, as shown in [KLDG05]).

In Section 2, where we assume Hypotheses (A-D), we study the homoclinic intersections for the perturbed Hamiltonian. We use the global map $S_{\varepsilon}: N_{ \pm}^{\mathrm{u}} \longrightarrow N_{ \pm}^{\mathrm{u}}$, defined by the flow of the Hamiltonian $H$, between the $(2 n+3)$ dimensional sections

$$
N_{ \pm}^{\mathrm{u}}=\left\{u_{1}= \pm d\right\}, \quad N_{ \pm}^{\mathrm{s}}=\left\{v_{1}= \pm d\right\}
$$

where the choice of the signs depends on the sections intersected by the loop $\gamma$. On these sections we have coordinates $\left(\varphi, \xi, v_{1}, u_{2}, v_{2}\right)$ and $\left(\varphi, \xi, u_{1}, u_{2}, v_{2}\right)$ respectively (we use the symplectic polar coordinates $(2)$ instead of $(x, y)$ ), 
but alternatively we can consider coordinates $\left(\varphi, \xi, c, u_{2}, v_{2}\right)$ in both sections, where $c$ is the energy level. For the unperturbed global map $S_{0}$, on a given energy level $c$ we can consider the $(2 n+2)$-dimensional reduced sections $N_{ \pm}^{\mathrm{s}, \mathrm{u}}(c)=N_{ \pm}^{\mathrm{s}, \mathrm{u}} \cap\left\{H^{(0)}=c\right\}$, both with symplectic coordinates $\left(x, y, u_{2}, v_{2}\right)$, and the restriction $S_{0}: N_{ \pm}^{\mathrm{u}}(c) \rightarrow N_{ \pm}^{\mathrm{u}}(c)$ is a symplectic map. Then, the map $S_{\varepsilon}$ is a perturbation of $S_{0}$, and can be described by a generating function $R_{\varepsilon}\left(\varphi, \bar{\xi}, \bar{u}_{2}, v_{2}\right)$.

The traces of the $(n+2)$-dimensional invariant manifolds $\mathcal{W}_{I, \varepsilon}^{\mathrm{s}, \mathrm{u}}$ in the sections $(7)$ are $(n+1)$-dimensional, and defined by

$$
\mathcal{A}_{I, \varepsilon}^{\mathrm{u}}=\mathcal{W}_{I, \varepsilon}^{\mathrm{u}} \cap N_{ \pm}^{\mathrm{u}}, \quad \mathcal{A}_{I, \varepsilon}^{\mathrm{s}}=\mathcal{W}_{I, \varepsilon}^{\mathrm{s}} \cap N_{ \pm}^{\mathrm{s}} \text {. }
$$

Such traces are contained in the same energy level $c$, and can be seen as cylinders (homeomorphic to $\mathbb{T}^{n} \times \mathbb{R}$ ), parameterized by $\left(\varphi, u_{2}\right)$ and $\left(\varphi, v_{2}\right)$ respectively. The intersections between the invariant manifolds $\mathcal{W}_{I, \varepsilon}^{\mathrm{s}, \mathrm{u}}$ are given by intersections between the sets $S_{\varepsilon}\left(\mathcal{A}_{I, \varepsilon}^{\mathrm{u}}\right)$ and $\mathcal{A}_{I, \varepsilon}^{\mathrm{s}}$, which can be studied in terms of the generating function $R_{\varepsilon}$, providing a generic condition for the existence of transverse homoclinic orbits (Theorem 1).

For the effective detection of transverse intersections in concrete Hamiltonians, it is standard to apply the Poincaré$M_{e l}^{\prime}$ nikov method. In view of the computations required by this method, it is hard to develop it in a general setting. For this reason, in Sections 3 and 4 we replace $(\mathbf{C}-\mathbf{D})$ by the following specific hypotheses:

$\left(\mathbf{C}_{2}\right)$ The 2-d.o.f. Hamiltonian $P$ is a product of 2 pendulums:

$$
\begin{aligned}
& P(q, p)=P_{1}\left(q_{1}, p_{1}\right)+P_{2}\left(q_{2}, p_{2}\right), \\
& P_{j}\left(q_{j}, p_{j}\right)=\frac{p_{j}^{2}}{2}+\lambda_{j}^{2}\left(\cos q_{j}-1\right)=\frac{p_{j}^{2}}{2}-2 \lambda_{j}^{2} \sin ^{2} \frac{q_{j}}{2}, \quad j=1,2
\end{aligned}
$$

(recall that $\lambda_{1}=1$ and $\lambda_{2}=\lambda$, with $1>\lambda$ ).

$\left(\mathbf{D}_{2}\right)$ The perturbation $H^{(1)}$ has the form

$$
H^{(1)}\left(x, y, q_{1}, q_{2}, p_{1}, p_{2}\right)=\left(\cos q_{1}-\nu\right) h\left(x, y, q_{2}, p_{2}\right),
$$

with $h=\mathcal{O}_{2}\left(x, y, q_{2}, p_{2}\right)$, and we may consider the cases $\nu=1$ or $\nu=0$.

We are considering in $\left(\mathbf{C}_{2}\right)$ a very concrete model for the hyperbolic part. It is integrable, with $K=P_{1}$ or $K=P_{2}$ as the second first integral. For this model, let us show that an accurate description of its loops can be given. It is well-known that each pendulum $P_{j}\left(q_{j}, p_{j}\right)$ has 2 separatrices, which are homoclinic orbits connecting the origin of $P_{j}$ to itself. Choosing one separatrix of each pendulum, we obtain 4 one-parameter families of homoclinic orbits of the Hamiltonian $P(q, p)$. The parameter of each family is the initial condition of one of the separatrices, which can be chosen freely. Additionally, there are 4 particular homoclinic orbits obtained from one separatrix of a pendulum and the origin of the other pendulum. The difference between these 4 orbits and the orbits of the 4 families lies in their different topology: in the configuration space $\mathbb{T}^{2}$, they describe cycles belonging to different homotopy classes. Among the 4 particular homoclinic orbits, notice that 2 of them are tangent at the origin to the strong stable/unstable directions (the pendulum $P_{1}$ ), and the other 2 ones are tangent to the weak stable/unstable directions (the pendulum $P_{2}$ ). Below, we take as our loop $\gamma$ one of the 'strong' ones. Clearly, if we consider the stable and unstable manifolds $W_{0}^{\mathrm{s}, \mathrm{u}}$ in a neighbourhood of the loop, they intersect transversely along the loop (inside the corresponding 3-dimensional energy level). The situation is different for a loop belonging to one of the 4 families, since the manifolds $W_{0}^{\mathrm{s}, \mathrm{u}}$ coincide in a neighbourhood of such a loop.

We stress that an analogous model to (9-10), with a higher number of degrees of freedom, has been considered in [RT06]. Another related work is [GS95], where the orbit considered is one from the 4 families of homoclinic orbits, but adding to $P_{1}+P_{2}$ an interacting term such that one specific orbit is preserved and becomes transverse. In fact, the existence of families of homoclinic orbits and the particular ones, is a more general property of 2-d.o.f. Hamiltonians having a 2-saddle (see [LU98, ch. 6] for a complete exposition).

The loop we consider is the one with $p_{1}>0$ and $q_{2}=p_{2}=0$, and is given by the trajectory

$$
\gamma: \quad\left(q_{1}^{\mathrm{h}}(t), q_{2}^{\mathrm{h}}(t), p_{1}^{\mathrm{h}}(t), p_{2}^{\mathrm{h}}(t)\right)=\left(4 \arctan \left(\mathrm{e}^{t}\right), 0, \frac{2}{\cosh t}, 0\right) .
$$


According to the results quoted about Hypothesis (C), the local normal form (6) can be considered for the Hamiltonian (9-10). In this case, this is a simple consequence of a well-known Moser's theorem [Mos56] on the convergence of the Birkhoff normal form for a 1-d.o.f. Hamiltonian near a hyperbolic equilibrium point. Indeed, this theorem can be applied separately to each pendulum $P_{j}\left(q_{j}, p_{j}\right)$, which can be written in the form $\widetilde{P}_{j}\left(\eta_{j}\right)=\lambda_{j} \eta_{j}+\Xi_{j}\left(\eta_{j}\right)$, and we have $\Xi\left(\eta_{1}, \eta_{2}\right)=\Xi_{1}\left(\eta_{1}\right)+\Xi_{2}\left(\eta_{2}\right)$ in $(6)$.

Alternatively, we can write the 2-d.o.f. Hamiltonian (9) keeping the original coordinates $\left(q_{1}, p_{1}\right)$ for the first pendulum, and using the normal form coordinates $\left(u_{2}, v_{2}\right)$ for the second pendulum, obtaining the expression $P_{1}\left(q_{1}, p_{1}\right)+$ $\widetilde{P}_{2}\left(\eta_{2}\right)$. Then the unstable manifold $W_{0}^{\mathrm{u}}$ is given by the equations $P_{1}\left(q_{1}, p_{1}\right)=0, v_{2}=0$, and the stable manifold $W_{0}^{\mathrm{s}}$ is given by $P_{1}\left(q_{1}, p_{1}\right)=0, u_{2}=0$. These invariant manifolds are local, but may be continued along a neighbourhood of the whole homoclinic orbit $\gamma$. It becomes also transparent that they intersect transversely along this orbit. Although this is a trivial fact, it may be surprising because the Hamiltonian (9) is clearly integrable. Besides, if we consider the invariant manifolds globally, they coincide since they are obtained as products of the separatrices of each pendulum. But in the case of invariant manifolds of a hyperbolic point, such a coincidence is not a contradiction with their local transversality, as noticed in [Dev78].

Finally, we have imposed in $\left(\mathbf{D}_{2}\right)$ a simplifying hypothesis for the perturbation $H^{(1)}$, similar to the case dealt with in [KLDG05]. It is easy to check that this hypothesis implies that, for $\varepsilon \neq 0$, both the loop $\gamma$ and the centre manifold $\mathcal{W}^{\mathrm{c}}$ are kept invariant. In the case $\nu=0$, we have a Cantor family $\mathcal{T}_{I, \varepsilon}$ of preserved KAM tori $\mathcal{T}_{I, \varepsilon}$ close to the unperturbed ones, as described above. In the case $\nu=1$, even the inner flow of $\mathcal{W}^{c}$ is kept invariant, and this implies that its invariant tori are all preserved, $\mathcal{T}_{I, \varepsilon}=\mathcal{T}_{I, 0}$, with the same frequencies.

For such preserved tori, using the Mel'nikov potential we show, in both cases $\nu=1$ and $\nu=0$, that the perturbed stable and unstable manifolds $\mathcal{W}_{I, \varepsilon}^{\mathrm{s}, \mathrm{u}}$ intersect along a finite number of transverse homoclinic orbits. The intersections will be associated to nondegenerate critical points of the Mel'nikov potential (Theorem 2). To give a definition of it, we consider unperturbed trajectories on both the invariant torus $\mathcal{T}_{I, 0}$ and the homoclinic cylinder $\mathcal{T}_{I, 0} \times \gamma$. Such trajectories are defined in Section 3: a trajectory on $\mathcal{T}_{I, 0}$ is denoted $\zeta_{I, 0}^{*}(t ; \theta)$ where $\theta \in \mathbb{T}^{n}$ corresponds to the initial phase of a trajectory, and a trajectory on $\mathcal{T}_{I, 0} \times \gamma$, that is asymptotic for $t \rightarrow \pm \infty$ to the previous trajectory on the torus, is denoted $\zeta_{I, 0}(t ; \theta)$. Then, we define the $M e l^{\prime}$ nikov potential as the following function on $\mathbb{T}^{n}$ :

$$
L_{I}(\theta)=-\int_{-\infty}^{\infty}\left(H^{(1)}\left(\zeta_{I, 0}(t ; \theta)\right)-H^{(1)}\left(\zeta_{I, 0}^{*}(t ; \theta)\right)\right) \mathrm{d} t .
$$

In Section 4 we compute the Mel'nikov potential and show that it can be approximated by a trigonometric polynomial that will be, generically, a Morse function. In this way, we can obtain (Theorem 3) a lower bound and an upper bound for the number of critical points and, consequently, transverse homoclinic orbits to a given torus.

\section{Study of the homoclinic orbits through the global map}

First, we consider $H^{(0)}$. According to Hypotheses $(\mathbf{A}-\mathbf{C})$ the unperturbed Hamiltonian casts $H^{(0)}(x, y, q, p)=$ $F(x, y)+P(p, q)$, where $F(x, y)$ is an integrable $n$-d.o.f, so it depends only on the actions $\xi$ and, on the other hand, $P$ is a two saddle with characteristic exponents \pm 1 and $\pm \lambda$ with $1>\lambda>0$. Therefore, in appropriate coordinates $(q, p)$ for the hyperbolic part $H^{(0)}$ can be expanded in the form

$$
H^{(0)}(x, y, q, p)=f(\xi)+q_{1} p_{1}+\lambda q_{2} p_{2}+\mathcal{O}_{3}(q, p)
$$

with $f(\xi)$ as given in (4), hypothesis (B). Because of the hypothesis $(\mathbf{C})$, linear independence of the quadratic parts of $P, P^{(2)}$, and $K^{(2)}$ of the additional integral $K$, we can apply the results of Vey in [Vey78] (see also [Ito89, theorem 1.3]), on the convergence of the Birkhoff normal form for locally integrable Hamiltonian systems; and hence conclude that there exists, defined in a neighbourhood of the origin, a near-identity analytic (convergent) Birkhoff transformation of type

$$
(q, p):=\phi(u, v)=(u, v)+\mathcal{O}_{2}(u, v)
$$

being $(q, p)=\left(q_{1}, q_{2}, p_{1}, p_{2}\right),(u, v)=\left(u_{1}, u_{2}, v_{2}, v_{2}\right)$ such that $\phi(0,0)=(0,0)$ and both the transformed Hamiltonian $\widetilde{P}(u, v)=P \circ \phi(u, v)$ and the additional integral $\widetilde{K}(u, v)=K \circ \phi(u, v)$ are analytic functions of the products $\eta_{1}:=u_{1} v_{1}$, $\eta_{2}:=u_{2} v_{2}$. In particular, this yields,

$$
\widetilde{P}(u, v)=\eta_{1}+\lambda \eta_{2}+\Xi\left(\eta_{1}, \eta_{2}\right)
$$


with $\Xi\left(\eta_{1}, \eta_{2}\right)=\mathcal{O}_{2}\left(\eta_{1}, \eta_{2}\right)$, in a neighbourhood the origin and $\eta_{1}, \eta_{2}$ are first integrals of the Hamiltonian $\widetilde{P}$.

Thus, we proceed now to investigate the unperturbed global map $S_{0}: N_{+}^{\mathrm{u}}(c) \rightarrow N_{ \pm}^{\mathrm{s}}(c)$. For this, we shall use Birkhoff local coordinates (13) and replace the coordinates $(x, y)$ by the polar symplectic ones $(\varphi, \xi)$. For a fixed level set $c$, of the Hamiltonian $H^{(0)}$, the equation

$$
f(\xi)+\eta_{1}+\lambda \eta_{2}+\Xi\left(\eta_{1}, \eta_{2}\right)=c,
$$

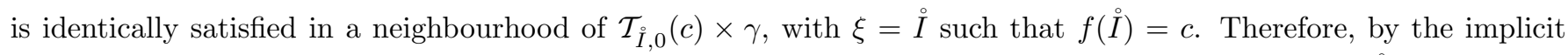
function theorem $\eta_{1}$ can be put as a function, $\kappa$, of $\xi, \eta_{2}$ in a neigbourhood of the point $\left(\xi, \eta_{1}, \eta_{2}\right)=(I, 0,0)$. Hence, for a fixed $c$ one can take $\left(\xi, \varphi, u_{2}, v_{2}\right)$ as coordinates on the $N_{+}^{u}(c)$. Indeed, since on this section $u_{1}=d$ and then $v_{1}=\kappa\left(\xi, \eta_{2} ; c\right) / d$. Let us fix a point $\left(\xi, \varphi, q_{2}, p_{2}\right) \in N_{+}^{u}(c)$ and denote as $T=\hat{\tau}\left(\xi, q_{2}, p_{2} ; c\right)$ the time needed to reach $N_{ \pm}^{s}(c)$. The global map $S_{0}: N_{+}^{\mathrm{u}}(c) \rightarrow N_{ \pm}^{\mathrm{s}}(c)$ is defined by the flow of the vector field $X_{H^{(0)}}$ in a (global) neighbourhood of the loop $\gamma$. On the one hand $\xi_{i}, i=1, \ldots, n$ are global integrals of $H^{(0)}$, so their values are preserved by $S_{0}$; and on the other, due to the hypothesis $(\mathbf{C})$, the local integrals $\eta_{1}, \eta_{2}$ are smooth functions of the (global) integrals $P, K$ in a vicinity of the origin and therefore, their values are preserved by $S_{0}$ as well. This has been used in setting (18), see also [KLDG05, Sect. 2].

Next, we look at the equations for the flow of $\widetilde{P}(u, v)$,

$$
\begin{array}{ll}
\dot{u}_{1}=\left(1+\Lambda_{1}\left(\eta_{1}, \eta_{2}\right)\right) u_{1}, & \dot{v}_{1}=-\left(1+\Lambda_{1}\left(\eta_{1}, \eta_{2}\right)\right) v_{1}, \\
\dot{u}_{2}=\left(\lambda+\Lambda_{2}\left(\eta_{1}, \eta_{2}\right)\right) u_{2}, & \dot{v}_{2}=-\left(\lambda+\Lambda_{2}\left(\eta_{1}, \eta_{2}\right)\right) v_{2},
\end{array}
$$

where $\Lambda_{1}\left(\eta_{1}, \eta_{2}\right)=\partial_{\eta_{1}} \Xi\left(\eta_{1}, \eta_{2}\right), \Lambda_{2}\left(\eta_{1}, \eta_{2}\right)=\partial_{\eta_{2}} \Xi\left(\eta_{1}, \eta_{2}\right)$.

The system above accounts for the flow of the unperturbed Hamiltonian on the hyperbolic directions, locally around the origin, and the explicit representation of its solutions are

$$
\begin{array}{ll}
\bar{u}_{1}=u_{1} \exp \left\{\left(1+\Lambda_{1}\left(\eta_{1}, \eta_{2}\right)\right) t\right\} & \bar{v}_{1}=v_{1} \exp \left\{-\left(1+\Lambda_{1}\left(\eta_{1}, \eta_{2}\right)\right) t\right\} \\
\bar{u}_{2}=u_{2} \exp \left\{\left(\lambda+\Lambda_{2}\left(\eta_{1}, \eta_{2}\right)\right) t\right\} & \bar{v}_{2}=v_{2} \exp \left\{-\left(\lambda+\Lambda_{2}\left(\eta 1, \eta_{2}\right)\right) t\right\}
\end{array}
$$

Let us denote now by $\mathcal{I}^{(0)}, \Theta^{(0)}$ and $\mathcal{Q}_{i}^{(0)}, \mathcal{P}_{i}^{(0)}, i=1,2$ the components of the solutions of $H^{(0)}$ in the elliptic and in the hyperbolic directions respectively, when the initial conditions are taken on $N_{+}^{u}(c)$. Therefore, the (unperturbed) map $S_{0}$ can be written as:

$$
S_{0}:\left(\begin{array}{c}
\varphi \\
\xi \\
q_{2} \\
p_{2}
\end{array}\right) \mapsto\left(\begin{array}{c}
\bar{\varphi} \\
\bar{\xi} \\
\bar{q}_{2} \\
\bar{p}_{2}
\end{array}\right)=\left(\begin{array}{c}
\Theta^{(0)}\left(\hat{\tau}\left(\xi, q_{2}, p_{2} ; c\right), \xi, q_{2}, p_{2} ; c\right) \\
\mathcal{I}^{(0)}\left(\hat{\tau}\left(\xi, q_{2}, p_{2} ; c\right), \xi, q_{2}, p_{2} ; c\right) \\
\mathcal{Q}_{2}^{(0)}\left(\hat{\tau}\left(\xi, q_{2}, p_{2} ; c\right), \xi, q_{2}, p_{2} ; c\right) \\
\mathcal{P}_{2}^{(0)}\left(\hat{\tau}\left(\xi, q_{2}, p_{2} ; c\right), \xi, q_{2}, p_{2} ; c\right)
\end{array}\right)=\left(\begin{array}{c}
D f(\xi) \hat{\tau}\left(\xi, q_{2}, p_{2} ; c\right)+\varphi \\
\xi \\
\mathcal{Q}_{2}^{(0)}\left(\hat{\tau}\left(\xi, q_{2}, p_{2} ; c\right), \xi, q_{2}, p_{2} ; c\right) \\
\mathcal{P}_{2}^{(0)}\left(\hat{\tau}\left(\xi, q_{2}, p_{2} ; c\right), \xi, q_{2}, p_{2} ; c\right)
\end{array}\right) .
$$

We stress that the first two components can be written explicitly, for the unperturbed Hamiltonian $H^{(0)}$ is separable in the elliptic and hyperbolic directions. Here, it has been taken into account the form of the Birkhoff transformation (13), that $u_{1}=d$ on $N_{+}^{u}(c), \bar{v}_{1}= \pm d$ on $N_{ \pm}^{s}(c)$ and that $\eta_{2}=u_{2} v_{2}=\bar{u}_{2} \bar{v}_{2}$. On the other hand, we note that close to $\mathcal{T}_{I, 0}(c) \times \gamma\left(\right.$ with $\left.\xi=\stackrel{\circ}{I} \in f^{-1}(c), u_{2}=v_{2}=0\right), \hat{\tau}(\stackrel{I}{I}, 0,0 ; c)=T_{0}$, the time taken for the homoclinic orbit $\gamma$ starting at $N_{+}^{u}(c)$ to meet $N_{ \pm}^{s}(c)$.

To construct $S_{0}$ thought of as a Poincaré map between two different transversal sections, namely $N_{+}^{u}(c)$ and $N_{ \pm}^{s}(c)$, we put together (16) and (17):

$$
S_{0}:\left(\begin{array}{c}
\varphi \\
\xi \\
u_{2} \\
v_{2}
\end{array}\right) \mapsto\left(\begin{array}{c}
\bar{\varphi} \\
\bar{\xi} \\
\bar{u}_{2} \\
\bar{v}_{2}
\end{array}\right)=\left(\begin{array}{c}
D f(\xi) \tau\left(\xi, u_{2}, v_{2} ; c\right)+\varphi \\
\xi \\
u_{2} \exp \left\{\left(\lambda+\Lambda_{2}\left(\eta_{1}, \eta_{2}\right)\right) \tau\left(\xi, u_{2}, v_{2} ; c\right)\right\} \\
v_{2} \exp \left\{-\left(\lambda+\Lambda_{2}\left(\eta_{1}, \eta_{2}\right)\right) \tau\left(\xi, u_{2}, v_{2} ; c\right)\right\}
\end{array}\right)
$$


where $\tau\left(\xi, u_{2}, v_{2} ; c\right)=\hat{\tau}\left(\xi, \phi^{-1}\left(u_{2}, v_{2}\right) ; c\right)$. Using the last two equations (18), we define the functions $G_{1}, G_{2}$ of $\xi, u_{2}, v_{2}, \bar{u}_{2}, \bar{v}_{2}$ :

$$
\begin{aligned}
& G_{1}\left(\xi, u_{2}, v_{2}, \bar{u}_{2}, \bar{v}_{2} ; c\right):=\bar{u}_{2}-u_{2} \exp \left\{\left(\lambda+\Lambda_{2}\left(\eta_{1}, \eta_{2}\right)\right) \tau\left(\xi, u_{2}, v_{2} ; c\right)\right\} \\
& G_{2}\left(\xi, u_{2}, v_{2}, \bar{u}_{2}, \bar{v}_{2} ; c\right):=\bar{v}_{2}-v_{2} \exp \left\{-\left(\lambda+\Lambda_{2}\left(\eta_{1}, \eta_{2}\right)\right) \tau\left(\xi, u_{2}, v_{2} ; c\right)\right\} .
\end{aligned}
$$

Therefore, from the equations $G_{1}\left(\xi, u_{2}, v_{2}, \bar{u}_{2}, \bar{v}_{2} ; c\right)=0$ and $G_{2}\left(\xi, u_{2}, v_{2}, \bar{u}_{2}, \bar{v}_{2} ; c\right)=0, u_{2}, \bar{v}_{2}$, can be casted as functions of $\bar{u}_{2}, v_{2}$, in a neighbourhood of $\left(\xi, u_{2}, v_{2}, \bar{u}_{2}, \bar{v}_{2}\right)=(\stackrel{\circ}{I}, 0,0,0,0)$, with $\stackrel{\Xi}{I} \in f^{-1}(c)$. To check this, one first obtains the corresponding Jacobian is then given by

$$
\frac{\partial\left(G_{1}, G_{2}\right)}{\partial\left(u_{2}, \bar{v}_{2}\right)}(\stackrel{\circ}{I}, 0,0,0,0 ; c)=\left|\begin{array}{cc}
-\exp \left\{\lambda T_{0}\right\} & 0 \\
0 & 1
\end{array}\right|
$$

which is different from zero since $T_{0}$ is a constant. Therefore, for a fixed $c$, there exists in a neighbourhood of $\left(\xi, u_{2}, v_{2}, \bar{u}_{2}, \bar{v}_{2}\right)=(\stackrel{\circ}{I}, 0,0,0,0)$ with $\xi=\stackrel{\circ}{I} \in f^{-1}(c)$ (and hence in a neighbourhood of $\left.\mathcal{T}_{I, 0}(c) \times \gamma, \stackrel{\circ}{I} \in f^{-1}(c)\right)$, where two functions $u_{2}, \bar{v}_{2}$ display as functions of $\xi, \bar{u}_{2}, v_{2}$, respectively: $u_{2}=\mathcal{U}_{0}\left(\xi, \bar{u}_{2}, v_{2} ; c\right), \bar{v}_{2}=\mathcal{V}_{0}\left(\xi, \bar{u}_{2}, v_{2} ; c\right)$. In this way, the global map for the unperturbed Hamiltonian, $H^{(0)}$ can be expressed through the form

$$
\tilde{S}_{0}:\left(\begin{array}{c}
\bar{\varphi} \\
\xi \\
u_{2} \\
\bar{v}_{2}
\end{array}\right) \mapsto\left(\begin{array}{c}
D f(\bar{\xi}) \widetilde{\tau}\left(\bar{\xi}, \bar{u}_{2}, v_{2} ; c\right)+\varphi \\
\bar{\xi} \\
\mathcal{U}_{0}\left(\xi, \bar{u}_{2}, v_{2} ; c\right) \\
\mathcal{V}_{0}\left(\xi, \bar{u}_{2}, v_{2} ; c\right)
\end{array}\right)
$$

where $\widetilde{\tau}\left(\bar{\xi}, \bar{u}_{2}, v_{2} ; c\right):=\tau\left(\bar{\xi}, \mathcal{U}_{0}\left(\xi, \bar{u}_{2}, v_{2} ; c\right), v_{2} ; c\right), c$ held fixed. Furthermore as the above is a symplectic map defined in a connected set, the 1-form: $\varpi=\bar{u}_{2} \mathrm{~d} \bar{v}_{2}+v_{2} \mathrm{~d} u_{2}+\bar{\varphi} \mathrm{d} \bar{\xi}+\xi \mathrm{d} \varphi$ must be closed. Hence, there exists a generating function, $R_{0}\left(\varphi, \bar{\xi}, \bar{u}_{2}, v_{2} ; c\right)$, such that

$$
\begin{array}{ll}
\bar{\varphi}=\frac{\partial R_{0}}{\partial \bar{\xi}}\left(\varphi, \bar{\xi}, \bar{u}_{2}, v_{2} ; c\right)=\varphi+D f(\bar{\xi}) \widetilde{\tau}\left(\bar{\xi}, u_{2}, \bar{v}_{2} ; c\right), & \xi=\frac{\partial R_{0}}{\partial \varphi}\left(\varphi, \bar{\xi}, \bar{u}_{2}, v_{2} ; c\right)=\bar{\xi}, \\
u_{2}=\frac{\partial R_{0}}{\partial \bar{v}_{2}}\left(\varphi, \bar{\xi}, \bar{u}_{2}, v_{2} ; c\right)=\mathcal{U}_{0}\left(\bar{\xi}, \bar{u}_{2}, v_{2} ; c\right), & \bar{v}_{2}=\frac{\partial R_{0}}{\partial u_{2}}\left(\varphi, \bar{\xi}, \bar{u}_{2}, v_{2} ; c\right)=\mathcal{V}_{0}\left(\bar{\xi}, \bar{u}_{2}, v_{2} ; c\right) .
\end{array}
$$

Then, the perturbed global map $\tilde{S_{\varepsilon}}$ has the generating function

$$
R_{\varepsilon}\left(\varphi, \bar{\xi}, \bar{u}_{2}, v_{2} ; c\right)=R_{0}\left(\varphi, \bar{\xi}, \bar{u}_{2}, v_{2} ; c\right)+\varepsilon R_{1}\left(\varphi, \bar{\xi}, \bar{u}_{2}, v_{2}, \varepsilon ; c\right) .
$$

We can change the coordinates in such a way that the traces $\mathcal{A}_{I, \varepsilon}^{\mathrm{s}, \mathrm{u}}$ of the torus $\mathcal{T}_{I, \varepsilon}$ on $N_{+}^{u}(c)$ and $N_{ \pm}^{s}(c)$ have the representations $\mathcal{A}_{I, \varepsilon}^{\mathrm{s}}=\left\{\bar{\xi}=I, \bar{u}_{2}=0\right\}$ and $\mathcal{A}_{I, \varepsilon}^{\mathrm{u}}=\left\{\xi=I, v_{2}=0\right\}$ (see [KLDG05] for details). A homoclinic orbit biasymptotic to the torus $\mathcal{T}_{I, \varepsilon}$ is given by an intersection between $S_{\varepsilon}\left(\mathcal{A}_{I, \varepsilon}^{\mathrm{u}}\right)$ and $\mathcal{A}_{I, \varepsilon}^{\mathrm{s}}$. To find such intersections we have to solve the system of equations,

$$
\bar{\varphi}=\frac{\partial R_{\varepsilon}}{\partial \bar{\xi}}(\varphi, I, 0,0 ; c), \quad I=\frac{\partial R_{\varepsilon}}{\partial \varphi}(\varphi, I, 0,0 ; c), \quad \bar{u}_{2}=\frac{\partial R_{\varepsilon}}{\partial v_{2}}(\varphi, I, 0,0 ; c), \quad v_{2}=\frac{\partial R_{\varepsilon}}{\partial \bar{u}_{2}}(\varphi, I, 0,0 ; c) .
$$

In fact, the intersection can be detected from the second equation above, namely, taken into account (23) we can write:

$$
0=\frac{\partial R_{1}}{\partial \varphi}(\varphi, I, 0,0, \varepsilon ; c),
$$

If we find a simple solution $\varphi_{0}^{*}(I ; c)$ of $(26)$ for $\varepsilon=0$, then the implicit function theorem can be applied to obtain a perturbed solution $\varphi_{\varepsilon}^{*}(I ; c)$ of $(25)$ for $\varepsilon$ small enough. Substituting it into other equations of $(25)$, we obtain the other coordinates of the solution. In this way, the function $R_{1}$ allows us to establish the main result of this section.

Theorem 1 For a given torus $\mathcal{T}_{I, \varepsilon}$, let $c=f(I)$ and assume that $R_{1}(\varphi, I, 0,0,0 ; c)$ is a Morse function on $\mathbb{T}^{n}$. Then, for $\varepsilon$ small enough there exist at least $2^{n+1}$ homoclinic orbits to $\mathcal{T}_{I, \varepsilon}$. 


\section{Remarks.}

1. In general, for a function of $n$ angles the minimum number of intersections is $2^{n}$, unless there are some symmetries as in our case owing to assumption (D). More precisely, this is discussed in Section 4.

2. A related result was obtained in [LMS03] using a different technique, that leads to a system of equations similar to (25). In that paper, some topological techniques were used in order to show the existence of intersections, since a gradient form is not as transparent as in (26).

3. To arrive at $(26)$, we had to assume $\varepsilon \neq 0$ since we divided by $\varepsilon$. Indeed, for $\varepsilon=0$ there is a degeneracy in the second equation of (25): in this case $\varphi \in \mathbb{T}^{n}$ can be chosen freely and we have a whole homoclinic cylinder (as described in Section 1).

\section{The Mel'nikov potential}

The arguments given in the previous section show that the existence of (transverse) homoclinic orbits seems to be, in principle, quite general. But it looks rather hard to apply those arguments to a given Hamiltonian. In order to detect the effective existence of transverse homoclinic orbits in a concrete case, and to obtain a first approximation for such orbits, the standard procedure is the Poincaré-Mel'nikov method. This method provides the (vector) Mel'nikov function as an approximation to the distance between the stable and unstable manifolds $\mathcal{W}_{I, \varepsilon}^{\mathrm{s}, \mathrm{u}}$ of a given torus $\mathcal{T}_{I, \varepsilon}$. A transverse homoclinic orbit corresponds to a simple zero of this function. Besides, the Hamiltonian character of the equations allows us to look for nondegenerate critical points of the (scalar) Mel'nikov potential, whose gradient is the $\mathrm{Mel}^{\prime}$ nikov function (see [DG00]).

For the sake of simplicity in the formulation of the method, we restrict ourselves to the case of an unperturbed Hamiltonian $H^{(0)}$ and a perturbation $H^{(1)}$, satisfying the hypotheses $\left(\mathbf{A}, \mathbf{B}, \mathbf{C}_{2}, \mathbf{D}_{2}\right)$ described in Section 1 .

It will be convenient to use the coordinates $\zeta=\left(\varphi, \xi, q_{1}, p_{1}, u_{2}, v_{2}\right)$. In this way, we replace $(x, y)$ by the symplectic polar coordinates $(\varphi, \xi)$ introduced in $(2)$; the reason for this is that the actions $\xi=\left(\xi_{1}, \ldots, \xi_{n}\right)$, which are first integrals of $H^{(0)}$, allow us to provide a first approximation for the distance. On the other hand, for the pendulum $P_{1}$ we keep the global coordinates $\left(q_{1}, p_{1}\right)$ because we take into account the whole loop $\gamma$. For the pendulum $P_{2}$ we replace $\left(q_{2}, p_{2}\right)$ by the local coordinates $\left(u_{2}, v_{2}\right)$, since we only need to consider a neighbourhood of the loop $\gamma$ in its transverse directions.

Recall that the unperturbed stable and unstable manifolds $\mathcal{W}_{I, 0}^{\mathrm{s}, \mathrm{u}}$ intersect along the homoclinic cylinder $\mathcal{T}_{I, 0} \times \gamma$, which is an $(n+1)$-dimensional manifold, and that they are both contained in a $(2 n+3)$-dimensional energy level. To measure the distance between the perturbed manifolds $\mathcal{W}_{I, \varepsilon}^{\mathrm{s}, \mathrm{u}}$, we consider $(2 n+3)-(n+1)=n+2$ directions, transverse to the homoclinic cylinder. The usual approach in the Poincaré-Mel'nikov method (see for instance [DG00]), is to consider a certain number first integrals of $H^{(0)}$ (as many as possible), independent on the energy level, and such that their gradients are transverse to the unperturbed intersection. We have $\xi_{1}, \ldots, \xi_{n}, P_{1}$ as suitable first integrals, but only $n$ of them are independent, say $\xi_{1}, \ldots, \xi_{n}$. The distance along the directions provided by such first integrals will be $\mathcal{O}(\varepsilon)$. The second pendulum $P_{2}=g_{2}\left(u_{2} v_{2}\right)$ is another first integral, but it is degenerate on the homoclinic cylinder. To complete the set of $n+2$ directions, we are going to consider the coordinates $u_{2}, v_{2}$, although they are not first integrals and the distance along the associated directions will be $\mathcal{O}(1)$.

Let us describe the parameterizations to be used. We consider a fixed Diophantine torus $\mathcal{T}_{I, \varepsilon}$ in the centre manifold $\mathcal{W}^{\mathrm{c}}$, with actions $I=\left(I_{1}, \ldots, I_{n}\right)$. This torus can be parameterized by $\theta=\left(\theta_{1}, \ldots, \theta_{n}\right) \in \mathbb{T}^{n}$ in the coordinates provided by KAM theorem (see Section 1). Let $\mathcal{Z}^{*}=\mathcal{Z}_{I, \varepsilon}^{*}(\theta)$ be the parameterization of the torus $\mathcal{T}_{I, \varepsilon}$ in the coordinates $\zeta$, and let us denote $\mathcal{I}^{*}=\left(\mathcal{I}_{1}^{*}, \ldots, \mathcal{I}_{n}^{*}\right)$ the $\xi$-components of $\mathcal{Z}^{*}$.

Recall from (8) that we denote $\mathcal{A}_{I, \varepsilon}^{\mathrm{u}}, \mathcal{A}_{I, \varepsilon}^{\mathrm{s}}$ the traces of the invariant manifolds $\mathcal{W}_{I, \varepsilon}^{\mathrm{u}}, \mathcal{W}_{I, \varepsilon}^{\mathrm{s}}$ on the transverse sections $N^{\mathrm{u}}=N_{+}^{\mathrm{u}}, N^{\mathrm{s}}=N_{ \pm}^{\mathrm{s}}$ respectively. The transverse sections $N^{\mathrm{s}, \mathrm{u}}$ have been defined in (7) by a number $d$, which can be chosen small enough (not depending on $\varepsilon$ ) in such a way that the traces are contained in the domain of validity of (6). 
As we showed, such traces can be considered as cylinders (homeomorphic to $\mathbb{T}^{n} \times \mathbb{R}$ ), which makes a difference with the case of 1 saddle, where such traces are just tori (homeomorphic to $\mathbb{T}^{n}$, see [KLDG05]).

For the trace $\mathcal{A}_{I, \varepsilon}^{\mathrm{u}}$, we consider a parameterization $\mathcal{Z}^{\mathrm{u}}=\mathcal{Z}_{I, \varepsilon}^{\mathrm{u}}\left(\theta, \hat{u}_{2}\right)$, with the parameters $\theta \in \mathbb{T}^{n}$ and small $\hat{u}_{2} \in \mathbb{R}$ (as in the unperturbed trace $\mathcal{A}_{I, 0}^{\mathrm{u}}$ ). The angles $\theta$ can be taken in such a way that the trajectory starting at a point $\mathcal{Z}_{I, \varepsilon}^{\mathrm{u}}\left(\theta, \hat{u}_{2}\right)$ is asymptotic for $t \rightarrow-\infty$ to the trajectory starting at $\mathcal{Z}_{I, \varepsilon}^{*}(\theta)$. Similarly, for the trace $\mathcal{A}_{I, \varepsilon}^{\mathrm{s}}$ we consider a parameterization $\mathcal{Z}^{\mathrm{s}}=\mathcal{Z}_{I, \varepsilon}^{\mathrm{s}}\left(\theta, \hat{v}_{2}\right)$, where the the trajectory starting at this point is asymptotic for $t \rightarrow \infty$ to the one starting at $\mathcal{Z}_{I, \varepsilon}^{*}(\theta)$. For the parameterizations $\mathcal{Z}^{\mathrm{s}, \mathrm{u}}$ of the traces, we consider their $\xi$-components $\mathcal{I}^{\mathrm{s}, \mathrm{u}}=\left(\mathcal{I}_{1}^{\mathrm{s}, \mathrm{u}}, \ldots, \mathcal{I}_{n}^{\mathrm{s}, \mathrm{u}}\right)$, and we are also interested in their $\left(u_{2}, v_{2}\right)$-components $\mathcal{U}_{2}^{\mathrm{s}, \mathrm{u}}, \mathcal{V}_{2}^{\mathrm{s}, \mathrm{u}}$. (It is clear, for the parameterization $\mathcal{Z}^{*}$ of the torus, that we have $\mathcal{U}_{2}^{*}=\mathcal{V}_{2}^{*}=0$.)

Now, we consider the image of $\mathcal{A}_{I, \varepsilon}^{\mathrm{u}}$ through the global map $S_{\varepsilon}: N^{\mathrm{u}} \rightarrow N^{\mathrm{s}}$, and denote $\overline{\mathcal{Z}}^{\mathrm{u}}=\overline{\mathcal{Z}}_{I, \varepsilon}^{\mathrm{u}}\left(\theta, \hat{u}_{2}\right)$ the parameterization of $S_{\varepsilon}\left(\mathcal{A}_{I, \varepsilon}^{\mathrm{u}}\right)$ inherited from $\mathcal{A}_{I, \varepsilon}^{\mathrm{u}}$, i.e. $\overline{\mathcal{Z}}^{\mathrm{u}}$ is the point where the trajectory starting at a point $\mathcal{Z}^{\mathrm{u}}$ intersects $N^{\mathrm{s}}$. As before, we denote $\overline{\mathcal{I}}^{\mathrm{u}}=\left(\overline{\mathcal{I}}_{1}^{\mathrm{u}}, \ldots, \overline{\mathcal{I}}_{n}^{\mathrm{u}}\right), \overline{\mathcal{U}}_{2}^{\mathrm{u}}, \overline{\mathcal{V}}_{2}^{\mathrm{u}}$ the $\left(\xi, u_{2}, v_{2}\right)$-components of the point $\overline{\mathcal{Z}}^{\mathrm{u}}$.

Our aim is to detect the transversality between the cylinders $S_{\varepsilon}\left(\mathcal{A}_{I, \varepsilon}^{\mathrm{u}}\right)$ and $\mathcal{A}_{I, \varepsilon}^{\mathrm{s}}$. To study this, we are going to take the distance, along the $\left(\xi, u_{2}, v_{2}\right)$-directions, between two points $\overline{\mathcal{Z}}^{\mathrm{u}}, \mathcal{Z}^{\mathrm{s}}$ belonging to those cylinders. In order to measure the distance correctly, the phase $\theta$ in the parameters of each point has to be chosen carefully.

First, we provide a suitable description of the trajectories in the unperturbed case $\varepsilon=0$. A trajectory of $H^{(0)}$ on $\mathcal{W}_{I, 0}^{\mathrm{u}}$ is given, in the coordinates $\left(\varphi, \xi, q_{1}, p_{1}, u_{2}, v_{2}\right)$, by

$$
\zeta_{I, 0}^{\mathrm{u}}\left(t ; \theta, \hat{u}_{2}\right)=\left(\theta+t \widetilde{\omega}_{I}, I, q_{1}^{\mathrm{h}}(t), \dot{q}_{1}^{\mathrm{h}}(t), u_{2}^{(0)}\left(t ; \hat{u}_{2}\right), 0\right),
$$

where we denote $\widetilde{\omega}_{I}=\widetilde{\omega}(I)$ as defined in (5), the function $q_{1}^{\mathrm{h}}(t)$ was introduced in (11), and we use the notation $\left(u_{2}^{(0)}\left(t ; \hat{u}_{2}\right), 0\right)$ for the trajectory of $g_{2}\left(u_{2} v_{2}\right)$ starting at $\left(\hat{u}_{2}, 0\right)$ (this trajectory tends to $(0,0)$ as $t \rightarrow-\infty$ with exponential rate). Analogously, a trajectory on $\mathcal{W}_{I, 0}^{\mathrm{s}}$ is given by

$$
\zeta_{I, 0}^{\mathrm{s}}\left(t ; \theta, \hat{v}_{2}\right)=\left(\theta+t \widetilde{\omega}_{I}, I, q_{1}^{\mathrm{h}}(t), \dot{q}_{1}^{\mathrm{h}}(t), 0, v_{2}^{(0)}\left(t ; \hat{v}_{2}\right)\right)
$$

where $\left(0, v_{2}^{(0)}\left(t ; \hat{v}_{2}\right)\right)$ denotes the trajectory of $g_{2}\left(u_{2} v_{2}\right)$ starting at $\left(0, \hat{v}_{2}\right)$ (it tends to $(0,0)$ as $t \rightarrow \infty$ with exponential rate).

Clearly, the trajectories (27-28) are asymptotic (with exponential estimates), for $t \rightarrow-\infty$ and $t \rightarrow \infty$ respectively, to the following trajectory on the unperturbed torus:

$$
\zeta_{I, 0}^{*}(t ; \theta)=\left(\theta+t \widetilde{\omega}_{I}, I, 0,0\right) .
$$

Notice also that the unperturbed trajectories with $\hat{u}_{2}=\hat{v}_{2}=0$ coincide:

$$
\zeta_{I, 0}(t ; \theta):=\zeta_{I, 0}^{\mathrm{u}}(t ; \theta, 0)=\zeta_{I, 0}^{\mathrm{s}}(t ; \theta, 0),
$$

becoming homoclinic trajectories, biasymptotic for $t \rightarrow \pm \infty$ to the same trajectory $\zeta_{I, 0}^{*}(t ; \theta)$. The union of such trajectories $\zeta_{I, 0}(t ; \theta)$ over $\theta \in \mathbb{T}^{n}$ fills the unperturbed homoclinic cylinder $\mathbb{T}^{n} \times \gamma$. (We point out that this is due to the fact that our unperturbed Hamiltonian $H^{(0)}$ in $(3)$ is separable or "uncoupled"; in a more general situation the coincidence of the asymptotics for $t \rightarrow \pm \infty$ does not take place, giving rise to a more complicated expression for the Mel'nikov potential; see [DG00].)

The trajectories $(27-28)$ satisfy, for $t=0$, the initial conditions $\zeta_{I, 0}^{\mathrm{u}}\left(0 ; \theta, \hat{u}_{2}\right)=\left(\theta, I, \pi, 0, \hat{u}_{2}, 0\right)$ and $\zeta_{I, 0}^{\mathrm{s}}\left(0 ; \theta, \hat{v}_{2}\right)=$ $\left(\theta, I, \pi, 0,0, \hat{v}_{2}\right)$. Let $T_{1}, T_{2}>0$ be such that $\zeta_{I, 0}^{\mathrm{u}}\left(-T_{1} ; \theta, \hat{u}_{2}\right) \in N^{\mathrm{u}}, \zeta_{I, 0}^{\mathrm{s}}\left(T_{2} ; \theta, \hat{v}_{2}\right) \in N^{\mathrm{s}}$. We have $T_{1}, T_{2} \sim \ln (1 / d)$, where $d$ is the number that defines the sections $N^{\mathrm{s}, \mathrm{u}}$ in $(7)$.

For the perturbed Hamiltonian, $\varepsilon \neq 0$, we are going to detect transverse intersections by comparing the $\left(\xi, u_{2}, v_{2}\right)$ components of two points $\overline{\mathcal{Z}}^{\mathrm{u}}$ and $\mathcal{Z}^{\mathrm{s}}$, both belonging to the section $N^{\mathrm{s}}$. The values of $T_{1}, T_{2}$ are taken into account in order to choose the phases of the points to be compared:

$$
\overline{\mathcal{Z}}_{I, \varepsilon}^{\mathrm{u}}\left(\theta-T_{1} \widetilde{\omega}_{I}, u_{2}^{(0)}\left(-T_{1} ; \hat{u}_{2}\right)\right) \quad \text { and } \quad \mathcal{Z}_{I, \varepsilon}^{\mathrm{s}}\left(\theta+T_{2} \widetilde{\omega}_{I}, v_{2}^{(0)}\left(T_{2} ; \hat{v}_{2}\right)\right)
$$


(notice that they coincide for $\varepsilon=0, \hat{u}_{2}=\hat{v}_{2}=0$, and any $\theta \in \mathbb{T}^{n}$ ). With that choice of the parameters, the transverse intersections will be given by simple zeros $\left(\theta^{*}, \hat{u}_{2}^{*}, \hat{v}_{2}^{*}\right)$ of the set of differences

$$
\overline{\mathcal{I}}^{\mathrm{u}}(\cdots)-\mathcal{I}^{\mathrm{s}}(\cdots), \quad \overline{\mathcal{U}}_{2}^{\mathrm{u}}(\cdots)-\mathcal{U}_{2}^{\mathrm{s}}(\cdots), \quad \overline{\mathcal{V}}_{2}^{\mathrm{u}}(\cdots)-\mathcal{V}_{2}^{\mathrm{s}}(\cdots)
$$

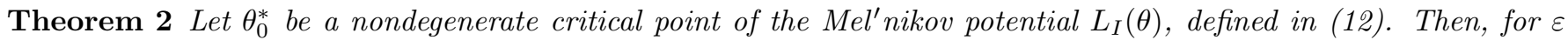
small enough there exists a transverse homoclinic orbit, given by a simple zero of the differences (29), of the form $\left(\theta^{*}, \hat{u}_{2}^{*}, \hat{v}_{2}^{*}\right)=\left(\theta_{0}^{*}, 0,0\right)+\mathcal{O}(\varepsilon)$

Proof. We use that the trajectories on the invariant manifolds $\mathcal{W}_{I, \varepsilon}^{\mathrm{s}, \mathrm{u}}$ are close to the unperturbed trajectories on $\mathcal{W}_{I, 0}^{\mathrm{s}, \mathrm{u}}$. For any $\theta \in \mathbb{T}^{n}$, and small $\hat{u}_{2}, \hat{v}_{2}$, let us consider the trajectories

$$
\zeta^{\mathrm{u}}(t):=\zeta_{I, \varepsilon}^{\mathrm{u}}\left(t ; \theta, \hat{u}_{2}\right), \quad \zeta^{\mathrm{s}}(t):=\zeta_{I, \varepsilon}^{\mathrm{s}}\left(t ; \theta, \hat{v}_{2}\right)
$$

satisfying the initial conditions

$$
\zeta^{\mathrm{u}}\left(-T_{1}\right)=\mathcal{Z}_{I, \varepsilon}^{\mathrm{u}}\left(\theta-T_{1} \widetilde{\omega}_{I}, u_{2}^{(0)}\left(-T_{1} ; \hat{u}_{2}\right)\right), \quad \zeta^{\mathrm{s}}\left(T_{2}\right)=\mathcal{Z}_{I, \varepsilon}^{\mathrm{s}}\left(\theta+T_{2} \widetilde{\omega}_{I}, v_{2}^{(0)}\left(T_{2} ; \hat{v}_{2}\right)\right) .
$$

The trajectory $\zeta^{\mathrm{u}}(t)$ is $\mathcal{O}(\varepsilon)$-close to $\zeta_{0}^{\mathrm{u}}(t):=\zeta_{I, 0}^{\mathrm{u}}\left(t ; \theta, \hat{u}_{2}\right)$ for $t \leq-T_{1}$. In an analogous way, the trajectory $\zeta^{\mathrm{s}}(t)$ is $\mathcal{O}(\varepsilon)$-close to $\zeta_{0}^{\mathrm{s}}(t):=\zeta_{I, 0}^{\mathrm{s}}\left(t ; \theta, \hat{v}_{2}\right)$ for $t \geq T_{2}$. Both trajectories $(30)$ are asymptotic, for $t \rightarrow-\infty$ and $t \rightarrow \infty$ respectively, to the trajectory $\zeta^{*}(t):=\zeta_{I, \varepsilon}^{*}(t ; \theta)$ on the torus $\mathcal{T}_{I, \varepsilon}$, starting at $\zeta^{*}\left(T_{2}\right)=\mathcal{Z}_{I, \varepsilon}^{*}\left(\theta+T_{2} \widetilde{\omega}_{I}\right)$; this trajectory is $\mathcal{O}(\varepsilon)$-close to $\zeta_{0}^{*}(t):=\zeta_{I, 0}^{*}(t ; \theta)$ for all $t$.

The estimate of $\zeta^{\mathrm{u}}(t)$ as $\mathcal{O}(\varepsilon)$-close to $\zeta_{0}(t)$ can also be extended to the interval $-T_{1} \leq t \leq T_{2}$, whose length is $\sim \ln (1 / d)$, not depending on $\varepsilon$ (see [KLDG05] for more details). Using this estimate, and the fact that the trajectory $\zeta^{\mathrm{u}}(t)$ intersects $N^{\mathrm{s}}$ transversely, we deduce that $\overline{\mathcal{Z}}^{\mathrm{u}}=\zeta^{\mathrm{u}}\left(T_{2}+\mathcal{O}(\varepsilon)\right)$ and, for the actions, $\overline{\mathcal{I}}^{\mathrm{u}}=\xi^{\mathrm{u}}\left(T_{2}\right)+\mathcal{O}\left(\varepsilon^{2}\right)$ since $\dot{\xi}=\mathcal{O}(\varepsilon)$.

Let us expand in $\varepsilon$ the $\left(\xi, u_{2}, v_{2}\right)$-components of the trajectories $\zeta^{\mathrm{u}}(t)$ and $\zeta^{\mathrm{s}}(t)$; these expansions are valid for $t \leq T_{2}$ and $t \geq T_{2}$ respectively:

$$
\begin{array}{rlrl}
\xi^{\mathrm{u}}(t) & =I+\varepsilon \xi^{\mathrm{u},(1)}(t)+\mathcal{O}\left(\varepsilon^{2}\right), & & \xi^{\mathrm{s}}(t)=I+\varepsilon \xi^{\mathrm{s},(1)}(t)+\mathcal{O}\left(\varepsilon^{2}\right), \\
u_{2}^{\mathrm{u}}(t) & =u_{2}^{(0)}(t)+\mathcal{O}(\varepsilon), & & u_{2}^{\mathrm{s}}(t)=\mathcal{O}(\varepsilon), \\
v_{2}^{\mathrm{u}}(t) & =\mathcal{O}(\varepsilon), & v_{2}^{\mathrm{s}}(t)=v_{2}^{(0)}(t)+\mathcal{O}(\varepsilon) .
\end{array}
$$

where we have written down only the terms that will be necessary. To simplify the notation, we have hidden the dependence on the parameters: $\xi^{\mathrm{u},(1)}(t):=\xi_{I}^{\mathrm{u},(1)}\left(t ; \theta, \hat{u}_{2}\right), \xi^{\mathrm{s},(1)}(t):=\xi_{I}^{\mathrm{s},(1)}\left(t ; \theta, \hat{v}_{2}\right), u_{2}^{(0)}(t):=u_{2}^{(0)}\left(t ; \hat{u}_{2}\right), v_{2}^{(0)}(t):=$ $v_{2}^{(0)}\left(t ; \hat{v}_{2}\right)$. Notice that the unperturbed terms in the expansions have been taken from (27-28). Recall that the unperturbed actions $\xi_{j}$ are all constant $\left(=I_{j}\right)$, because they are first integrals of the unperturbed Hamiltonian $H^{(0)}$, whereas the "hyperbolic" coordinates $u_{2}, v_{2}$ are not first integrals.

The differences (29) are given by

$$
\begin{aligned}
& \overline{\mathcal{I}}^{\mathrm{u}}(\cdots)-\mathcal{I}^{\mathrm{s}}(\cdots)=\xi^{\mathrm{u}}\left(T_{2}\right)-\xi^{\mathrm{s}}\left(T_{2}\right)+\mathcal{O}\left(\varepsilon^{2}\right)=\varepsilon\left(\xi^{\mathrm{u},(1)}\left(T_{2}\right)-\xi^{\mathrm{s},(1)}\left(T_{2}\right)\right)+\mathcal{O}\left(\varepsilon^{2}\right), \\
& \overline{\mathcal{U}}_{2}^{\mathrm{u}}(\cdots)-\mathcal{U}_{2}^{\mathrm{s}}(\cdots)=u_{2}^{\mathrm{u}}\left(T_{2}\right)-u_{2}^{\mathrm{s}}\left(T_{2}\right)+\mathcal{O}(\varepsilon)=u_{2}^{(0)}\left(T_{2}\right)+\mathcal{O}(\varepsilon), \\
& \overline{\mathcal{V}}_{2}^{\mathrm{u}}(\cdots)-\mathcal{V}_{2}^{\mathrm{s}}(\cdots)=v_{2}^{\mathrm{u}}\left(T_{2}\right)-v_{2}^{\mathrm{s}}\left(T_{2}\right)+\mathcal{O}(\varepsilon)=-v_{2}^{(0)}\left(T_{2}\right)+\mathcal{O}(\varepsilon) .
\end{aligned}
$$

We see from the implicit function theorem that, in the two equations $\overline{\mathcal{U}}_{2}^{\mathrm{u}}-\mathcal{U}_{2}^{\mathrm{s}}=\overline{\mathcal{V}}_{2}^{\mathrm{u}}-\mathcal{V}_{2}^{\mathrm{s}}=0$, we can solve

$$
\hat{u}_{2}=\tilde{u}_{2}(\theta ; I, \varepsilon)=\mathcal{O}(\varepsilon), \quad \hat{v}_{2}=\tilde{v}_{2}(\theta ; I, \varepsilon)=\mathcal{O}(\varepsilon)
$$

Replacing such solutions into the equation $\overline{\mathcal{I}}^{\mathrm{u}}-\mathcal{I}^{\mathrm{s}}=0$, the transverse intersections are given, in a first approximation, by the simple zeros of the (vector) $M e l^{\prime}$ nikov function

$$
M_{I}(\theta)=\xi_{I}^{\mathrm{u},(1)}\left(T_{2} ; \theta, 0\right)-\xi_{I}^{\mathrm{s},(1)}\left(T_{2} ; \theta, 0\right) .
$$


A standard argument shows that the function $M_{I}(\theta)$ can be expressed in terms of the perturbation $H_{1}$. To deduce the formula, we consider the trajectories $\zeta^{\mathrm{s}, \mathrm{u}}(t)=\zeta_{I, \varepsilon}^{\mathrm{s}, \mathrm{u}}(t ; \theta, 0)$ defined in $(30-31)$, with $\hat{u}_{2}=\hat{v}_{2}=0$. We see from the Hamiltonian equations for $H=H^{(0)}+\varepsilon H^{(1)}$ that $\dot{\xi}^{\mathrm{u}}=-\varepsilon \partial_{\varphi} H\left(\zeta^{\mathrm{u}}\right)$ and, expanding this equality in $\varepsilon$ and taking the term of degree 1, we obtain: $\dot{\xi}^{\mathrm{u},(1)}=-\partial_{\varphi} H^{(1)}\left(\zeta_{0}^{\mathrm{u}}\right)$, and similarly for $\xi^{\mathrm{s},(1)}$. Since we consider $\hat{u}_{2}=\hat{v}_{2}=0$, we have $\zeta_{0}^{\mathrm{u}}=\zeta_{0}^{\mathrm{s}}=\zeta_{0}$. Then, we can obtain $\xi^{\mathrm{u},(1)}\left(T_{2}\right)$ and $\xi^{\mathrm{u},(1)}\left(T_{2}\right)$ as simple integrals, taken over the intervals $t \leq T_{2}$ and $t \geq T_{2}$ respectively. In general these integrals are not absolutely convergent, but this difficulty can be overcome using that both trajectories $\zeta^{\mathrm{s}, \mathrm{u}}(t)$ are asymptotic, with an exponential bound, to the trajectory $\zeta^{*}(t)$ on the torus $\mathcal{T}_{I, \varepsilon}$ (see [DG00]). As before, we can expand in $\varepsilon$ this trajectory, and we obtain absolutely convergent integrals:

$$
\begin{aligned}
& \xi^{\mathrm{u},(1)}\left(T_{2}\right)-\xi^{*,(1)}\left(T_{2}\right)=-\varepsilon \int_{-\infty}^{T_{2}}\left(\partial_{\varphi} H^{(1)}\left(\zeta_{0}(t)\right)-\partial_{\varphi} H^{(1)}\left(\zeta_{0}^{*}(t)\right)\right) \mathrm{d} t, \\
& \xi^{\mathrm{s},(1)}\left(T_{2}\right)-\xi^{*,(1)}\left(T_{2}\right)=\varepsilon \int_{T_{2}}^{\infty}\left(\partial_{\varphi} H^{(1)}\left(\zeta_{0}(t)\right)-\partial_{\varphi} H^{(1)}\left(\zeta_{0}^{*}(t)\right)\right) \mathrm{d} t .
\end{aligned}
$$

Substracting the two integrals we obtain the Mel'nikov function $M_{I}(\theta)$ as a single integral, which is clearly the gradient of the Mel'nikov potential $L_{I}(\theta)$ defined in (12). Each nondegenate critical point $\theta^{*}$ of $L_{I}(\theta)$ gives rise, for $\varepsilon$ small enough, to a simple zero $\left(\theta^{*}, \hat{u}_{2}^{*}, \hat{v}_{2}^{*}\right)$ of the differences $(29)$.

Remark. The difficulty about the absolute convergence does not take place if we consider $\nu=1$ in Hypothesis $\left(\mathbf{D}_{2}\right)$. Indeed, in this case the perturbation $H^{(1)}$ vanishes on the unperturbed tori $\mathcal{T}_{I, 0}$, and we have a simpler expression for the Mel'nikov potential in (12).

\section{On the number of transverse homoclinic orbits}

As we showed in Section 3, the nondegenerate critical points $\theta^{*}$ of the $\operatorname{Mel}^{\prime}$ nikov potential $L_{I}(\theta)$ give rise to transverse homoclinic orbits of the torus $\mathcal{T}_{I, \varepsilon}$ for $\varepsilon$ small enough. We are going to show that, under Hypothesis $\left(\mathbf{D}_{2}\right)$ on the perturbation $H^{(1)}$, the Mel'nikov potential (12) can easily be computed and, generically, it will be a Morse function on $\mathbb{T}^{n}$, In such a case, we also provide lower and upper bounds for the number of critical points.

Let $\hat{h}\left(\varphi, \xi, u_{2}, v_{2}\right)$ denote the function $h$, expressed in the coordinates $\zeta$ used in Section 3 . Using that $\cos q_{1}^{h}(t)-1=$ $-2 / \cosh ^{2} t$, we get from Hypothesis $\left(\mathbf{D}_{2}\right)$ the following expression for the Mel'nikov potential:

$$
L_{I}(\theta)=2 \int_{-\infty}^{\infty} \frac{\hat{h}\left(\theta+t \widetilde{\omega}_{I}, I, 0,0\right)}{\cosh ^{2} t} \mathrm{~d} t
$$

(not depending on the value of $\nu$ ). To compute this integral, it is standard to consider the Fourier expansion in $\theta$. For any $k \in \mathbb{Z}^{n}$, we have

$$
\int_{-\infty}^{\infty} \frac{\cos \left\langle k, \theta+t \widetilde{\omega}_{I}\right\rangle}{\cosh ^{2} t} \mathrm{~d} t=\mathcal{J}_{\left\langle k, \widetilde{\omega}_{I}\right\rangle} \cos \langle k, \theta\rangle, \quad \int_{-\infty}^{\infty} \frac{\sin \left\langle k, \theta+t \widetilde{\omega}_{I}\right\rangle}{\cosh ^{2} t} \mathrm{~d} t=\mathcal{J}_{\left\langle k, \widetilde{\omega}_{I}\right\rangle} \sin \langle k, \theta\rangle,
$$

where, by residue theory,

$$
\mathcal{J}_{a}=\int_{-\infty}^{\infty} \frac{\cos a t}{\cosh ^{2} t} \mathrm{~d} t=\frac{\pi a}{\sinh (\pi a / 2)}, \quad a \neq 0 ; \quad \mathcal{J}_{0}=2
$$

Let us consider the expansion of $h$ in homogeneous polynomials, $h(x, y, 0,0)=\sum_{m \geq 2} h^{(m)}(x, y)$, starting at order 2 by our hypotheses. Let us write each homogeneous part in symplectic the symplectic polar coordinates $(2): h^{(m)}(x, y)=$ $\hat{h}^{(m)}(\varphi, \xi)$; this is a trigonometric polynomial of degree $\leq m$ in $\varphi$, with coefficients being homogeneous polynomials of degree $m$ in $\sqrt{\xi_{1}}, \ldots, \sqrt{\xi_{n}}$. By the previous formulas, we see that we can consider an expansion of the same type for the Mel'nikov potential, $L_{I}(\theta)=\sum_{m \geq 2} L_{I}^{(m)}(\theta)$, with each $\hat{L}^{(m)}(\theta)$ being a trigonometric polynomial of degree $\leq m$ in $\theta$, with coefficients being homogeneous polynomials of degree $m$ in $\sqrt{I_{1}}, \ldots, \sqrt{I_{n}}$. 
This says that, for small $|I|$, the $\mathrm{Mel}^{\prime}$ nikov potential $L_{I}(\theta)$ is dominated by the terms in $L_{I}^{(2)}(\theta)$. For a given action $I$, this is a trigonometric polynomial of degree $\leq 2$ in $\theta$.

The argument will be an extension of the one given in [KLDG05] for the case of only 2 angles. Let us consider the terms of order 2 in $h$, grouped in the following way:

$$
h^{(2)}(x, y)=\sum_{1 \leq j \leq l \leq n}\left(a_{j l} x_{j} x_{l}+b_{j l} x_{j} y_{l}+c_{j l} y_{j} x_{l}+d_{j l} y_{j} y_{l}\right)
$$

(for $j=l$, we consider $c_{j j}=0$ ). In the symplectic polar coordinates, this function becomes

$$
\begin{aligned}
\hat{h}^{(2)}(\varphi, \xi)= & \sum_{1 \leq j \leq n} \xi_{j}\left(\left(d_{j j}-a_{j j}\right) \cos 2 \varphi_{j}+b_{j j} \sin 2 \varphi_{j}+\left(a_{j j}+d_{j j}\right)\right) \\
& +\sum_{1 \leq j<l \leq n} \sqrt{\xi_{j} \xi_{l}}\left(\left(d_{j l}-a_{j l}\right) \cos \left(\varphi_{j}+\varphi_{l}\right)+\left(b_{j l}+c_{j l}\right) \sin \left(\varphi_{j}+\varphi_{l}\right)\right. \\
& \left.+\left(a_{j l}+d_{j l}\right) \cos \left(\varphi_{j}-\varphi_{l}\right)+\left(b_{j l}-c_{j l}\right) \sin \left(\varphi_{j}-\varphi_{l}\right)\right) .
\end{aligned}
$$

Using the formulas (32-33), we can compute the part of order 2 of the Mel'nikov potential:

$$
\begin{aligned}
L_{I}^{(2)}(\theta)= & 2 \sum_{1 \leq j \leq n} I_{j}\left(\left(d_{j j}-a_{j j}\right) \mathcal{J}_{2 \omega_{j}} \cos 2 \theta_{j}+b_{j j} \mathcal{J}_{2 \omega_{j}} \sin 2 \theta_{j}+\left(a_{j j}+d_{j j}\right) \mathcal{J}_{0}\right) \\
& +2 \sum_{1 \leq j<l \leq n} \sqrt{I_{j} I_{l}}\left(\left(d_{j l}-a_{j l}\right) \mathcal{J}_{\omega_{j}+\omega_{l}} \cos \left(\theta_{j}+\theta_{l}\right)+\left(b_{j l}+c_{j l}\right) \mathcal{J}_{\omega_{j}+\omega_{l}} \sin \left(\theta_{j}+\theta_{l}\right)\right. \\
& \left.+\left(a_{j l}+d_{j l}\right) \mathcal{J}_{\omega_{j}-\omega_{l}} \cos \left(\theta_{j}-\theta_{l}\right)+\left(b_{j l}-c_{j l}\right) \mathcal{J}_{\omega_{j}-\omega_{l}} \sin \left(\theta_{j}-\theta_{l}\right)\right)
\end{aligned}
$$

where we have replaced $\left\langle k, \widetilde{\omega}_{I}\right\rangle$ by $\langle k, \omega\rangle$, since the part depending on $I$ goes to the terms $L_{I}^{(m)}(\theta)$ with $m>2$.

For any fixed $I=\left(I_{1}, \ldots, I_{n}\right)$, we have to study for nondegenerate critical points of $L_{I}^{(2)}(\theta)$. We have a homogeneous trigonometric polynomial of degree 2 (plus constant terms $\left(a_{j j}+d_{j j}\right) \mathcal{J}_{0}$ that do not need to take into account). This homogeneous polynomial is of generic type if we assume that $I_{1}, \ldots, I_{n}>0$. Its critical points depend on $I_{1}, \ldots, I_{n}$, but they are constant along the rays $I_{j} / I_{n}=$ const, $j=1, \ldots, n-1$. Generically, a homogeneous trigonometric polynomial of degree 2 is a Morse function, i.e. its critical points are all nondegenerate. We are going to provide lower and upper bounds for the number of critical points. Such nondegenerate critical points constitute an approximation for the critical points of the whole Mel'nikov potential $L_{I}(\theta)$, and are in correspondence with transverse homoclinic orbits.

Theorem 3 Let $\mathcal{B}$ be the set of actions $I$ such that $L_{I}^{(2)}(\theta)$ is a Morse function of $\theta \in \mathbb{T}^{n}$. Then, for $I$ in an open subset of $\mathcal{B}$, and $\varepsilon$ small enough, the torus $\mathcal{T}_{I, \varepsilon}$ has transverse homoclinic orbits, whose number is even, between $2^{\text {n+1 }}$ and $2^{2 n}$.

Proof. For a Morse function on $\mathbb{T}^{n}$, a well-known result from Morse theory [Mil63] says that there are at least $2^{n}$ critical points. However, in the case under consideration, the function $\mathcal{K}(\theta):=L_{I}^{(2)}(\theta)$ (a homogeneous trigonometric polynomial of degree 2 plus a constant) has a symmetry, which implies that the minimum number of critical points will be higher. Namely, we see from (34)

that the map on $\mathbb{T}^{n}$ defined by

$$
S:\left(\theta_{1}, \ldots, \theta_{n}\right) \longmapsto\left(\theta_{1}+\pi, \ldots, \theta_{n}+\pi\right)
$$

satisfies $\mathcal{K} \circ S=\mathcal{K}$. In view of this, we carry out the linear change on $\mathbb{T}^{n}$ defined by

$$
\psi_{j}=\theta_{j}-\theta_{j+1}, \quad j=1, \ldots, n-1, \quad \psi_{n}=\theta_{n}
$$


(it is one-to-one, since it is defined by a matrix with determinant $=1$ ), and consider our function expressed in the new coordinates: $\mathcal{K}(\theta)=\widetilde{\mathcal{K}}(\psi)$. Then, the symmetry $S$ becomes

$$
\widetilde{S}:\left(\psi_{1}, \ldots, \psi_{n-1}, \psi_{n}\right) \longmapsto\left(\psi_{1}, \ldots, \psi_{n-1}, \psi_{n}+\pi\right),
$$

i.e. only one coordinate is concerned. Thus, the function $\widetilde{\mathcal{K}}(\psi)$ is $2 \pi$-periodic in $\psi_{1} \ldots, \psi_{n-1}$, but $\pi$-periodic in $\psi_{n}$. In other words, we have two copies of a "reduced" function, and we have at least $2^{n} \cdot 2=2^{n+1}$ critical points.

In order to provide an upper bound for the number of critical points, we use algebraic arguments. In fact, we are going to obtain a somewhat more general result: if a homogeneous trigonometric polynomial $\mathcal{K}(\theta)$, of degree $m$, is a Morse function on $\mathbb{T}^{n}$, then it has at most $(2 m)^{n}$ critical points. To prove this, we consider the system of $n$ equations

$$
\partial_{\theta_{j}} \mathcal{K}\left(\theta_{1}, \ldots, \theta_{n}\right)=0, \quad j=1, \ldots, n,
$$

whose solutions are the critical points. The left hand sides are homogeneous trigonometric polynomials of degree $m$ (with zero mean, i.e. no constant terms). Let us denote $x_{j}=\sin \theta_{j}, y_{j}=\cos \theta_{j}$ (we can imagine this as coming back to the original coordinates of the $n$ centres). Then, the previous equations turn into $n$ polynomial equations, homogeneous of degree $m$. Adding the identities $\sin ^{2} \theta_{j}+\cos ^{2} \theta_{j}-1=0$, we obtain a system of $2 n$ equations:

$$
P_{j}\left(x_{1}, y_{1}, \ldots, x_{n}, y_{n}\right)=0, \quad x_{j}^{2}+y_{j}^{2}-1=0, \quad j=1, \ldots, n,
$$

Introducing homogeneous coordinates $x_{j}=X_{j} / Z, y_{j}=Y_{j} / Z$, the system of equations becomes

$$
P_{j}\left(X_{1}, Y_{1}, \ldots, X_{n}, Y_{n}\right)=0, \quad Q_{j}\left(X_{j}, Y_{j}, Z\right)=X_{j}^{2}+Y_{j}^{2}-Z^{2}=0, \quad j=1, \ldots, n .
$$

Applying Bézout's theorem [Sha94, p. 236] to this system, one can establish that the number of solutions is either infinite, or equal to the product of the degrees of the equations: $(2 m)^{n}$. This number includes both real and complex solutions (counted with multiplicity), as well as solutions at infinity. Notice that all real solutions correspond to the critical points of $\mathcal{K}(\theta)$, since solutions at infinity $(Z=0)$, if they occur, are all complex. To exclude the possibility of an infinite number of solutions, notice that in such a case some pair of polynomials in (36) would have a common factor. It is clear that $Q_{j}, Q_{l}, j \neq l$, have no common factor. Also, since the variable $Z$ does not come into $P_{j}$, no common factors exist between $P_{j}$ and $Q_{l}$, for any $j, l$. Finally, suppose that $P_{j}, P_{l}, j \neq l$, have a common factor: $P_{j}=R_{j} F, P_{l}=R_{l} F$. Since these polynomials have real coefficients, we may assume that $F$ is a real common factor (if they have a complex common factor $G$, then $F=G \bar{G}$ would be a real common factor). Then, real solutions with $F=0$ would not be isolated, contradicting the nondegeneracy of the critical points. Otherwise, if there are not real solutions with $F=0$, we could replace $P_{j}, P_{l}$ by $R_{j}, R_{l}$ in the system (36), obtaining in this way a new system with the same real solutions, but no common factors. For this new system, the number of solutions provided by Bézout's theorem would be finite.

In this way, we have an upper bound for the number of critical points. In our case $m=2$, this upper bound is $(2 m)^{n}=2^{2 n}$. Recall that we also have a lower bound $2^{n+1}$. Among the solutions of the system (36), we have to exclude complex solutions, and solutions at infinity (which are also complex). Since complex solutions come in conjugated pairs, we have an even number of critical points.

Once we have established the possible number of critical points of $L_{I}^{(2)}(\theta)=\mathcal{K}(\theta)$, we have to extend this result to the whole $\mathrm{Mel}^{\prime}$ nikov potential $L_{I}(\theta)$. Recall that the coefficients of $L_{I}^{(2)}(\theta)$ are homogeneous polynomials of degree 2 in $\sqrt{I_{1}}, \ldots, \sqrt{I_{n}}$. This says that, if we consider the dependence of the critical points on $I=\left(I_{1}, \ldots, I_{n}\right)$, they are constant along the rays $I_{j} / I_{n}=r_{j}, j=1, \ldots, n-1$, and the concrete rays where the Morse condition is not fulfilled have to be excluded. For $|I|$ small enough, we can see $L_{I}(\theta)=L_{I}^{(2)}(\theta)+\mathcal{O}\left(|I|^{3 / 2}\right)$ as a perturbation and it will be also a Morse function, whose critical points tend to constant along each ray $I_{j} / I_{n}=r_{j}$, as $|I| \rightarrow 0$. In this case, we have to exclude a small neighbourhood of the rays $I_{j} / I_{n}=r_{j}$ where the Morse condition fails, and consider the union of the remaining rays. Finally, to obtain the set $\mathcal{B}$ of the theorem we have to select the actions $I$ with Diophantine frequencies $\widetilde{\omega}_{I}$. For the surviving perturbed tori $\mathcal{T}_{I, \varepsilon}$, we have as many transverse homoclinic orbits as the number of critical points of the Mel'nikov potential.

\section{Remarks.}

1. The case $n=1$ (i.e. only 1 centre) is much simpler and one directly obtains from the expression of $L_{I}^{(2)}(\theta)$ that the number of transverse homoclinic orbits is exactly 4 . 
2. For the case $n=2$, a constructive proof, but much longer, was provided in [KLDG05], obtaining either 8,12 or 16 transverse homoclinic orbits.

3. For any value of $n$, we established that the number of critical points is even, using that the complex solutions of (36) come in conjugated pairs. In fact, the complex solutions not at infinity come in groups of 4 , due to the conjugacy and to the symmetry (35), though this symmetry does not apply to the (complex) solutions at infinity. However, in general such solutions at infinity will not occur (they have to satisfy $Z=0$ as an additional equation to (36)), and the number of critical points would be a multiple of 4 .

\section{Acknowledgments}

The authors AD, PG and JRP were supported in part by the Spanish MCyT-FEDER grant MTM2006-00478, MICINN-FEDER grant MTM2009-06973, and the Catalan CUR-DIUE grant 2009SGR859. Besides, JRP was also supported by MICINN-PN $(\mathrm{I}+\mathrm{D}+\mathrm{I})$ 2008-2011 (grant JC2009-00306). The author OK is thankful for partial financial support from the Russian Foundation of Basic Research (grants 07-01-00715a, 08-01-00083a) and Marie Curie International Incoming Fellowship (grant 221004).

\section{References}

[Dev78] Robert L. Devaney. Transversal homoclinic orbits in an integrable system. Amer. J. Math., 100(3):631-642, 1978.

[DG00] A. Delshams and P. Gutiérrez. Splitting potential and the Poincaré-Mel'nikov method for whiskered tori in Hamiltonian systems. J. Nonlinear Sci., 10(4):433-476, 2000.

[GS95] V.G. Gelfreich and D.K. Sharomov. Examples of Hamiltonian systems with transversal homoclinic orbits. Phys. Lett. A, 197(2):139-146, 1995.

[Ito89] Hidekazu Ito. Convergence of Birkhoff normal forms for integrable systems. Comment. Math. Helv., 64(3):412-461, 1989.

[KLDG05] Oksana Koltsova, Lev Lerman, Amadeu Delshams, and Pere Gutiérrez. Homoclinic orbits to invariant tori near a homoclinic orbit to center-center-saddle equilibrium. Phys. D, 201(3-4):268-290, 2005.

[LMS03] P. Lochak, J.-P. Marco, and D. Sauzin. On the splitting of invariant manifolds in multidimensional near-integrable Hamiltonian systems. Mem. Amer. Math. Soc., 163(775):viii+145, 2003.

[LU98] L. M. Lerman and Ya. L. Umanskiy. Four-dimensional integrable Hamiltonian systems with simple singular points (topological aspects), volume 176 of Translations of Mathematical Monographs. American Mathematical Society, Providence, RI, 1998. Trans. from the Russian manuscript by A. Kononenko and A. Semenovich.

[Mil63] J. Milnor. Morse theory. Based on lecture notes by M. Spivak and R. Wells. Annals of Mathematics Studies, No. 51. Princeton University Press, Princeton, N.J., 1963.

[Mos56] Jürgen Moser. The analytic invariants of an area-preserving mapping near a hyperbolic fixed point. Comm. Pure Appl. Math., 9:673-692, 1956.

[Pös82] Jürgen Pöschel. Integrability of Hamiltonian systems on Cantor sets. Comm. Pure Appl. Math., 35(5):653-696, 1982.

[RT06] M. Rudnev and V. Ten. A model for separatrix splitting near multiple resonances. Regul. Chaotic Dyn., 11(1):83-102, 2006 .

[Sha94] Igor R. Shafarevich. Basic algebraic geometry. 1. Springer-Verlag, Berlin, second edition, 1994. Varieties in projective space, Translated from the 1988 Russian edition and with notes by Miles Reid.

[Vey78] J. Vey. Sur certains systèmes dynamiques séparables. Amer. J. Math., 100(3):591-614, 1978. 